\title{
Franziska Martinsen
}

\section{Gerechtigkeit für Betroffene!?}

\section{Völkerrechtliche Strafgerechtigkeit revisited}

\section{Gerechtigkeit durch Strafe - auch im Völkerstrafrecht eine „offene Frage"1}

Die Etablierung eines ständigen Internationalen Strafgerichtshofs (IStGH) im Jahre 2002 bedeutete unbestritten einen großen Fortschritt hinsichtlich der Ahndung sog. völkerrechtlicher Kernverbrechen, d.h. des Völkermords, des Verbrechens gegen die Menschlichkeit, des Kriegsverbrechens und (ab 2017) des Verbrechens der Aggression. Der IStGH entstand bekanntlich nicht aus dem Nichts, ihm gingen die Internationalen Militärgerichtstribunale von Nürnberg und Tokio, vor allem aber die beiden Ad-hoc-Gerichtshöfe für das ehemalige Jugoslawien (JStGH) und Ruanda (RStGH) voraus, in denen in Reaktion auf humanitäre Verbrechen während bewaffneter Konflikte bereits eine transnationale Rechtsprechung erfolgte. Anknüpfend an deren Errungenschaften bezüglich der Präzisierung und Differenzierung des Völkerstrafrechts ist die Errichtung des IStGH als beachtenswerter Schritt sowohl bei der Manifestation als auch der Weiterentwicklung einer internationalen Jurisdiktion zu werten. Das Ziel des Völkerstrafrechts besteht gemäß der Präambel des Römischen Statuts vornehmlich im Schutz der kollektiven Rechtsgüter Frieden, Sicherheit und Wohl der Welt. Zugleich rückt jedoch auch im Völkerstrafrecht - wie im Völkerrecht insgesamt - die Bedeutung des Menschenrechtsschutzes ${ }^{2}$ zunehmend in den Mittelpunkt: Im Zuge der internationalen Rechtsprechung wird das Leid von Personen, die im Kontext von Kernverbrechen zu Geschädigten werden, öffentlich thematisch. So wird die Verletzung des Individuums bei der Beschreibung der Straftatbestände $\mathrm{e}^{3}$ ausdrücklich benannt und nicht mehr stillschweigend als mehr oder weniger notwendige Begleiterscheinung von kriegerischen Auseinandersetzungen betrachtet. Dass das heutige Völkerstrafrecht die Verletzung völkerrechtlicher Normen durch Einzelpersonen unter Strafe stellt und den Täter_innen somit individuelle Verantwortlichkeit zuweist, ist dabei als richtungweisend zu werten. Grundsätzlich stellt sich allerdings die Frage, mit welchem Zweck überhaupt die Bestrafung für derart schwerwiegende Verbrechenstaten begründet werden kann, scheinen doch weitestgehend alle üblichen Strafzwecke, absolute wie relative, angesichts der Schwere und des Ausmaßes der „Menschheitsverbrechen “4 versagen zu müssen. ${ }^{5}$ „Vergangenheitsbewältigung“" wird neuerdings als möglicher Strafzweck genannt, er ist allerdings der klassischen Straftheorie unbekannt und darüber hinaus stark umstritten. ${ }^{6}$

1 Vgl. Reese, Fünf offene Fragen zum Internationalen Strafgerichtshof - und der Versuch einer Antwort, in: Neubacher/Klein (Hrsg.), Vom Recht der Macht zur Macht des Rechts? Interdisziplinäre Beiträge zur Zukunft internationaler Strafgerichte, Berlin 2006, 71-89 (71); Neubacher, Strafzwecke und Völkerstrafrecht, NJW 2006, 966-970 (966).

2 Vgl. Möller, Völkerstrafrecht und Internationaler Strafgerichtshof. Kriminologische, straftheoretische und rechtspolitische Aspekte, Münster 2003, 419 f.; Klindt, Menschenrechte und Souveränität, Berlin 2007, 70 f., Schulz, Transitional Justice und hybride Gerichte, Berlin 2009, $57 \mathrm{f}$.

3 Vgl. Art. 6-8 Römisches Statut.

4 Jäger, Menschheitsverbrechen und die Grenzen des Kriminalitätskonzeptes, KritV 76 (1993), 259-275.

5 Vgl. Reuss, ,Vergangenheitsbewältigung' als Strafzweck bei Völkerverbrechen?, Einsicht03, 2010, 34-43 (35); vgl. auch seinen Beitrag in diesem Heft.

6 Vgl. Schulz (Fn. 2), 13 f.; kritisch: Möller (Fn. 2), 426 ff. 
Angesichts des 10jährigen Jubiläums des Inkrafttretens des Römischen Statuts besteht daher das Anliegen dieses Aufsatzes in einer Untersuchung, inwiefern Betroffenen von schweren Menschenrechtsverletzungen durch eine völkerstrafrechtliche Jurisdiktion, deren Fokus auf der Bestrafung von Täter_innen liegt, Gerechtigkeit widerfahren kann. Hierzu wird zunächst zu erläutern sein, welche Strafgerechtigkeitskonzeption dem Völkerstrafrecht zugrunde liegt und ob diese die Belange von Betroffenen schwerwiegender Menschenrechtsverletzungen zu berücksichtigen vermag. Wie in der nationalen Rechtsprechung lässt sich auch auf internationaler Ebene eine zunehmende Hinwendung zum Opfer ${ }^{7}$ in der Strafjustiz feststellen. ${ }^{8}$ Jedoch wird die Einbeziehung von Geschädigtenbelangen in die Strafrechtstheorie nicht unkontrovers diskutiert. Dass die Partizipation von Betroffenen im Rahmen von Gerichtsverfahren vor dem IStGH per Statut vorgesehen ist und die Beteiligung von Betroffenen damit über die Rolle als Zeug_innen hinausgeht, wird in etlichen Publikationen jüngeren Datums explizit affirmierend betrachtet, insbesondere hinsichtlich der Berücksichtigung spezifischer Bedürfnisse von Betroffenen, z.B. nach Wiedergutmachung und Entschädigung. ${ }^{9}$ Der Befürwortung einer verstärkten Berücksichtigung von Betroffenenbelangen liegt in erster Linie die Auffassung zugrunde, dass ein gerechtigkeitstheoretisch gerechtfertigter Anspruch seitens der Betroffenen besteht, sich gegenüber offiziellen Institutionen Gehör zu verschaffen und das erfahrene individuelle Leid öffentlich zu machen. Hier geht es um die Beachtung des Bedürfnisses von Betroffenen nach Anerkennung ihrer Unrechtserlebnisse und der besonderen Schwere der Verletzungen, die sie im Rahmen eines Gerichtsverfahrens sowohl - zumindest stellvertretend - von politischen Verantwortlichen als auch von den Täter_innen, die ihnen das konkrete Unrecht angetan haben, einfordern können sollen. Darüber hinaus ist der Beteiligung von Betroffenen in internationalen Strafprozessen auch insofern besonderer Stellenwert zuzumessen, als sie wesentlich zur Legitimation des Völkerstrafrechts nicht nur in der Vor-Ort-Öffentlichkeit von Postkonfliktgesellschaften, sondern vor allem innerhalb der globalen Zivilgesellschaft, beiträgt. ${ }^{10}$ Allerdings birgt der normative Anspruch, mittels des Strafzwecks der Vergangenheitsbewältigung die Bedürfnisse von Betroffenen befriedigen zu können, andererseits die Gefahr einer Überforderung des Völkerstrafrechts. Inwiefern ein alternatives Verständnis der Aufarbeitung begangenen Unrechts zur Ausgestaltung einer an den Betroffenenbelangen orientierten, umfassenderen Gerechtigkeitskonzeption, die sowobl juridische als auch zivilgesellschaftliche Akteur_innen und Institutionen einbezieht, beitragen kann, wird im Schlussteil des Aufsatzes aufgezeigt.

\section{Sinn und Zweck völkerrechtlicher Bestrafung}

Angesichts der gravierenden Ausmaße der Kernverbrechen steht die herkömmliche Strafzwecktheorie vor der grundsätzlichen Frage, ob „Menschheitsverbre-

7 Aufgrund der problematischen Konnotation des Begriffs „Opfer“, insb. der implizierten Reproduktion einer Rolle der Passivität und Wehrlosigkeit, wird im Folgenden der Terminus „Betroffene/Betroffener“ bzw. „Geschädigte/Geschädigter“ verwendet. Je nach Kontext ist die Bezeichnung „Opfer“ jedoch unumgänglich, um die inhaltliche Bedeutung des Umstands, Opfer - im Gegensatz zu Täter_in - zu sein, zu verdeutlichen. Vgl. Hamel, Strafen als Sprechakt. Die Bedeutung der Strafe für das Opfer, Berlin 2009, 13, sowie Hassemer/Reemtsma, Verbrechensopfer. Gesetz und Gerechtigkeit, München 2002, 53.

8 Vgl. Hassemer/Reemtsma (Fn. 7), insb. Kap. 1 u. Kap. 3.II.; Hamel (Fn. 7), 13 f.

9 Vgl. exemplarisch: deBrouwer, Reparation to Victims of Sexual Violence, LJIL 20 (2007), 207-237; Hoven, Opfer im Völkerstrafrecht, ZStW 122 (2010), 706-722; Doak, The Therapeutic Dimension of Transitional Justice: Emotional Repair and Victim Satisfaction in International Trials and Truth Commissions, ICLR 11 (2011), 263-298.

10 Vgl. Glasius, What is Global Justice and Who Decides? Civil Society and Victim Responses to the International Criminal Court's First Investigations, Human Rights Quarterly 31 (2009), 496-520. 
chen“ mit derselben Strafgerechtigkeitskonzeption beantwortet werden können wie ,gewöhnliche' Kriminalität ${ }^{11}$ - hinsichtlich der besonderen Schwere der Menschenrechtsverletzungen erscheint es als regelrecht fraglich, inwiefern dieser Art von Unrechtstaten überhaupt angemessen zu begegnen ist. Die Begründung von Strafgerechtigkeit orientiert sich prinzipiell an der Idee der auch moralischen Gültigkeit einer Rechtsordnung im Ganzen. Die Dringlichkeit, Verstöße gegen diese Rechts- bzw. Gerechtigkeitsordnung zu ahnden, kann entsprechend damit plausibilisiert werden, dass eine Gesellschaft, die sich der Verurteilung einer Verbrechenstat enthält, sich zur Komplizin des Unrechts macht. ${ }^{12}$ Strafgerechtigkeit wird damit legitimiert, dass die Ahndung einer Unrechtstat die Gerechtigkeitsordnung, die die Basis einer an den Prinzipien der Rechtsstaatlichkeit orientierten Gesellschaft ist, (wieder)herstellt. Vor dem Hintergrund von Konflikt- bzw. Postkonfliktgesellschaften stellt sich die zentrale Resozialisierungsidee der relativen Strafzwecklehre jedoch ungleich komplexer dar als in auf weitestgehend verlässlichen moralischen Werten beruhenden, funktionsfähigen Rechtsstaaten. Dies liegt in der „besonderen Natur“13 der Menschheitsverbrechen begründet, bei der sich nicht nur Schweregrad und Ausmaß der Verbrechen von Delikten in friedlichen und stabilen Gesellschaften unterscheiden. Kernverbrechen werden vornehmlich unter von Konflikthaftigkeit, Unsicherheit, Verrohung und Gewaltförmigkeit geprägten gesellschaftlichen Umständen begangen. Die makrodelinquente Tat ist kein isoliertes Ereignis, sondern wird innerhalb eines kollektiven Aktionszusammenhangs verübt, bei dem oftmals staatliche bzw. militärische Akteur_innen in Form von Initiierung, Verstärkung oder Duldung der Konflikte beteiligt sind. ${ }^{14} \mathrm{Da}$ die Rückfallgefahr von Täter_innen makrokrimineller Verbrechen gemeinhin als gering eingeschätzt wird, sobald die Konfliktsituation beendet ist, wird argumentiert, dass das Völkerstrafrecht nicht auf die straftheoretische Rechtfertigung der notwendigen individuellen Abschreckung gestützt werden kann. Das „Denkzettelmodell“ als Komponente der Spezialpräventionstheorie sei damit für das Völkerstrafrecht insgesamt als ungeeignet zu betrachten. ${ }^{15}$ In Bezug auf die Bestimmung des Strafzwecks mag diese Einschätzung zwar stimmen, hinsichtlich der gesamtgesellschaftlichen Situation vor, während und nach der Konfliktphase ist jedoch der Umstand zu problematisieren, dass die makrokriminellen Unrechtstaten in fast allen Fällen bestehende gesellschaftliche Strukturen, insbesondere Geschlechterstereotypen, die bereits zu Friedenszeiten präsent sind, reproduzieren und durch deren Perpetuierung verstärken. ${ }^{16}$ Betroffene erleben oftmals insbesondere in der Postkonfliktphase ein regelrechtes „Kontinuum“17 der Gewalt, das mit einem allgemeinen Klima der Verrohung, Repression und Verhärtung gesellschaftlicher Rollenbilder einhergeht. Mit Blick auf die Lage von Betroffenen sind bei der Frage nach dem Sinn einer auf Prävention ausgerichteten Strafe daher nicht nur der individuelle Resozialisierungsaspekt der/des Täter_in, sondern vor dem Hintergrund der soziopolitischen Gesamtsituation einer Postkonfliktgesellschaft zudem die Erfolgsaussichten einer Aufarbeitung der Gewalttaten durch die Gesamtgesellschaft in Betracht zu ziehen.

Vgl. Bock, Das Opfer vor dem Internationalen Strafgericht, Berlin 2010, 166 ff.; Jäger (Fn. 4), 121; Möller (Fn. 2), 243. 


\section{Strafgerechtigkeit im Völkerstrafrecht}

Das Völkerstrafrecht vereint Aspekte des Völkerrechts (Rechtsquelle ist das Internationale Recht) und des Strafrechts (individuelles Verhalten wird unter Strafe gestellt) und bezieht seine Legitimation aus sämtlichen völkerrechtlichen Verträgen (u.a. den Statuten Internationaler Tribunale) und dem Völkergewohnheitsrecht (u.a. der Haager Landkriegsordnung von 1907, den Genfer Abkommen) sowie aus von den Kulturvölkern anerkannten allgemeinen Rechtsgrundsätzen. ${ }^{18}$ Die Definition der Kernverbrechen der Artikel 6-8 des Römischen Statuts beruht z.B. auf bestehenden völkerrechtlichen Konventionen: Art. 6 (Völkermord) hat das Abkommen über die Verhütung und Bestrafung des Völkermords von 1948, ${ }^{19}$ Art. 7 (Verbrechen gegen die Menschlichkeit) u.a. die Statute der Ad-hoc-Strafgerichtshöfe JStGH ${ }^{20}$ und $\mathrm{RStGH}^{21}$ zur Grundlage. Art. 8 (Kriegsverbrechen) basiert im Wesentlichen auf dem Humanitären Völkerrecht. Der Sinn völkerrechtlicher Strafgerechtigkeit liegt nach geltender Auffassung vornehmlich in der Bekräftigung der Normgeltung. Durch Aussprache eines Unrechturteils erfolgt sowohl eine Missbilligung der Verletzung geltenden Völkerrechts sowie der Menschenrechte als auch die Affirmation der Gültigkeit völker- und menschenrechtlicher Normen dadurch, dass der/dem Täter_in persönliche Verantwortung für deren Verletzung zugeschrieben wird. Primär schützt das Völkerstrafrecht supraindividuelle bzw. kollektive Rechtsgüter (vgl. Präambel Abs. 3 Römisches Statut). Zugleich schützt es jedoch auch individuelle Rechtsgüter. Der Völkermordtatbestand beispielsweise bezieht sich nicht nur auf die Verletzungen, die eine Gruppe von Betroffenen erleidet, sondern ebenso auf die Verletzung der Menschenwürde, des Lebens oder der körperlichen Unversehrtheit einzelner Geschädigter. ${ }^{22}$

Im Zentrum des Gesamtspektrums internationalen Strafrechts steht der Anspruch, den Spezifika von Kernverbrechen gerecht zu werden. Dieses Anliegen ist von drei Ambivalenzen gekennzeichnet. Die erste Ambivalenz besteht darin, dass makrokriminielle Taten aufgrund ihres Schweregrads und ihres spezifischen Kollektivtatzusammenhangs innerhalb einer Konfliktsituation nicht als „normale“ Kriminalität aufgefasst, im Zuge der Kriminalisierung aber explizit in den „ordinären“ Bestandteil strafrechtlich zu begegnender Kriminalität eingeordnet werden. ${ }^{23}$ Die Feststellung individueller Verantwortlichkeit für die makrokriminelle Tat bildet daher einen der Schwerpunkte des IStGH-Statuts. Damit wird jedoch eine zweite Ambivalenz berührt: Während „alltägliche“ Kriminalität eine Abweichung von der rechtlichen Ordnung darstellt, werden viele makrokriminelle Taten von den Täter_innen im Bewusstsein einer gewissen Konformität gegenüber (jedoch oftmals nur vermeintlich) temporär gültigen Normen während der Konfliktphase begangen. Eine dritte Ambivalenz liegt schließlich im „Opfer“-Begriff des Völkerstrafrechts. Dieses befasst sich nur mit Fällen, in denen Individuen als Angehörige einer bestimmten Gruppe oder Gemeinschaft Betroffene von Verbrechen sind. Es werden zwar die Verbrechenstaten gegen Einzelne bestraft, aber nur insofern, als sie im Kollektivtatzusammenhang stehen. Dies lässt sich anhand der Tatbestände ablesen, die im IStGH-Statut unter die Völkerstrafrechtsverbrechen subsumiert werden. Völkermord etwa wird defi-

Vgl. Art. 38 Abs. 1a-c Römisches Statut.

Vgl. BGBl 1954 II S. 729.

Vgl. S/25704 vom 3.5.1993.

Vgl. S/RES/955 vom 8.11.1994.

Vgl. Ambos, Möglichkeiten und Grenzen völkerstrafrechtlichen Rechtsgüterschutzes, in: Neubacher/ Klein (Hrsg.) (Fn. 1), 111-116 (111/112).

Vgl. Möller (Fn. 2), 522. 
niert als Handlung, die in der Absicht begangen wird, eine nationale, ethnische, rassische oder religiöse Gruppe zu vernichten (vgl. Art. 6 Römisches Statut). Einerseits wird mit dem Augenmerk auf die einzelne geschädigte Person der individuelle Menschenrechtsschutz hervorgehoben, andererseits das Opfer selbst überhaupt nur als Teil einer bestimmten Gruppe als Betroffene/Betroffener wahrgenommen. Da die Straftatbestände des Völkerrechts immer auch dem Schutz kollektiver Rechtsgüter dienen und das Römische Statut nur Angriffe auf einzelne Individuen, die in einem übergeordneten Kontext erfolgen, pönalisiert, ${ }^{24}$ entsteht das gerechtigkeitstheoretische Problem, dass das individuelle Verbrechensopfer nicht in erster Linie um seiner selbst willen berücksichtigt wird, sondern dass seine individuellen, menschenrechtsbasierten Ansprüche auf Gerechtigkeit abhängig von der Konfliktgesamtsituation gemacht werden.

\section{Ziele einer völkerrechtlichen Strafgerechtigkeit}

War die Rechtsprechung der Militärgerichtshöfe von Nürnberg und Tokio noch fast ausschließlich auf Vergeltung und Schuldausgleich ausgerichtet, so orientierte sich die Mehrheit der Urteile des JStGH und des RStGH am Strafzweck der positiven Generalprävention. Die Präambel des Römischen Statuts benennt als zentrale Ziele des Völkerstrafrechts die Beendigung der Straflosigkeit von Täter_innen sowie die Verhütung von Kernverbrechen. Erstere wird als positivgeneralpräventive Absicht gedeutet, letztere lässt sich als negativ-präventiver Strafzweck auffassen. Hier bleibt allerdings abzuwarten, welche Argumentation innerhalb der Rechtsprechung des IStGH sich etablieren wird. In Bezug auf die internationale Strafgerichtsbarkeit steht die Strafzwecktheorie vor der Frage, inwiefern nationale Strafzwecke direkt in die internationale Rechtsprechung übertragen werden können. Im Einzelnen beruft sich die internationale Rechtsprechung auf z.T. sehr unterschiedliche Strafzwecke, deren Begründung jedoch jeweils mit der entsprechenden globalen Bedeutung in allen Rechtssystemen angegeben wird.

Auch wenn die Strafzwecke im Römischen Statut nicht eindeutig definiert sind, geht ein verbreitetes Verständnis davon aus, dass das Statut des IStGH ebenfalls eine präventive Wirkung vorsieht. ${ }^{25}$ Die Präambel des Römischen Statuts gibt allgemein als Strafzwecke die Beendigung von Straflosigkeit und Prävention an: Erklärtes Ziel ist es, „der Straflosigkeit der Täter ein Ende zu setzten und so zur Verhütung solcher Verbrechen beizutragen“.26 Das Ziel der Beendigung der Straflosigkeit individuell verantwortlicher Täter_innen wird zumeist so verstanden, dass die Verletzung eines internationalen Rechtsgutes von der Staatengemeinschaft sanktioniert werden muss. Zugleich wird davon aber auch eine Signalwirkung gegenüber potentiellen Täter_innen erwartet. Ob „Verhütung“ somit mit „Abschreckung“ oder eher mit einer Form der Sozialprävention gleichgesetzt werden kann, ist nicht eindeutig. Im letzteren Falle ließe sich der Strafzweck im Sinne einer modifiziert positiven Generalprävention als ,Erziehung durch Stigmatisierung' fassen. Die Bestrafung der/des Täter_in dient nach diesem Verständnis einer „edukativen Systemprävention“27 vordringlich dazu, Unrechtstaten demonstrativ zu kriminalisieren - auch mit dem Ziel, die bislang eher rhetorisch verbleibende Menschenrechtsthematik anhand der völkerstrafrechtlichen 
Ahndung als durchsetzbares Recht aufzuwerten. Zugleich soll das allgemeine Rechtsempfinden in der Bevölkerung gestärkt und die Nicht-Anerkennungswürdigkeit makrokrimineller Taten bekräftigt werden. Problematisch bleibt bei diesem Strafzweckverständnis allerdings, dass die Verurteilungen von Täter_innen schauprozesshaften Charakter tragen können, der zu einer Viktimisierung einzelner Täter_innen führen kann. Außerdem verbindet sich damit in praktischer Hinsicht die Gefahr einer Ungleichbehandlung gegenüber all denjenigen (meist ressourcenstarken, ranghohen oder einflussreichen) Täter_innen, die nicht vor Gericht gestellt werden.

\section{Die Berücksichtigung von Geschädigteninteressen im Römischen Statut}

Ein gravierendes Problem herkömmlicher relativer Strafzwecktheorien besteht in Bezug auf die Betroffenen von Verbrechenstaten generell darin, dass sie mit ihrem dezidiert auf die Zukunft gerichteten präventionsorientierten Ansatz die geschädigte Person, die eine Unrechtstat konkret in der Vergangenheit erlitten hat, nicht genügend zu berücksichtigen vermögen. ${ }^{28}$ Diesem Defizit versuchen neuere Ansätze der Viktimologie, die darauf abzielen, die Interessen von Verbrechensopfern sowohl in die Urteilsfindung als auch bereits in den Strafprozess selbst einzubeziehen, zu begegnen. Sowohl auf nationaler wie internationaler Ebene wird das Verbrechensopfer längst nicht mehr vornehmlich als Lieferant_in prozessrelevanter Informationen angesehen, sondern es erhält zunehmend aktive Einflussmöglichkeiten auf das Prozessgeschehen. ${ }^{29}$ Grundlage für diesen Perspektivwechsel ist nicht nur eine zugleich umfassendere und differenziertere Wahrnehmung subjektiver Unrechtserlebnisse und Erfahrungen der Betroffenheit von Opfern schwerwiegender Menschenrechtsverletzung, die nicht zuletzt im Rahmen der Arbeit der Sondertribunale eine Sensibilisierung seitens der Mitglieder der Gerichte erfuhr.

Auch die Öffentlichkeitsarbeit von Nichtregierungsorganisationen und Aktivist_innen - zu nennen wäre hier bspw. das zivilgesellschaftliche Menschenrechtstribunal von Tokio ${ }^{30}$ - hat die zunehmende Hinwendung zu den Belangen von Betroffenen befördert. Eine entscheidende Rolle spielen vor allem neuere Ansätze der Strafzwecklehre, die die Berücksichtigung der Perspektive von Geschädigten mit klassischen Strafzwecken zu verbinden bzw. neue Strafzwecke z.B. den der Vergangenheitsbewältigung - zu definieren versuchen. Die Bedürfnisse und Erwartungen von Betroffenen variieren nicht nur in Abhängigkeit der Tat, der Tatfolgen, der Persönlichkeit der/des Geschädigten, des spezifischen kulturellen Hintergrunds sowie des jeweiligen politischen Konfliktzusammenhangs, sondern sind prinzipiell als dynamisch anzusehen. Die Überlegungen zur Integration von Betroffenenbelangen in die Strafgerechtigkeitskonzeption des Völkerstrafrechts orientieren sich daher eher an allgemeinen und grundlegenden Kriterien, die bei der Erörterung der Frage nach dem Wirkungspotential der Bestrafung der Täter_in für die Betroffenen von Belang sein könnten. Der Beendigung der Straflosigkeit kommt hierbei eine zentrale Bedeutung zu, da die Bestrafung durch offizielle Institutionen (sei es auf der Grundlage eines nationalen oder supranationalen Gerichtsurteils) nicht nur als Bestätigung der Erwartungen an das geltende Recht, sondern auch als wichtige Form der Anerkennung der Unrechtserfahrung und des persönlichen Leids der Geschädigten angesehen wird. 
Aufgrund seiner normbekräftigenden Zielsetzung erscheint der Strafzweck der

Generalprävention, insbesondere in seiner Ausrichtung auf eine edukative Systemprävention, als am ehesten geeignet, den Interessen von Betroffenen entgegenzukommen. So verbindet sich insbesondere mit der positiven Generalprävention für die Geschädigten die Erwartung, dass von offizieller Seite aus mittels Verhängung einer Strafe ein explizites öffentliches Brandmarken der Tat durch die Internationale Gemeinschaft erfolgt, die im günstigsten Fall mit der Erfahrung der Betroffenen, dass Recht sich gegen Unrecht durchsetzt, zur Deckung kommt. $^{31}$

\section{Betroffenengerechte Strafverfabren}

Hinsichtlich der Berücksichtigung von Betroffenenbelangen wird neben der Strafe für die/den Urheber_in der Unrechtstat als dem Ergebnis des Strafprozesses bereits dem Strafprozess selbst besondere Aufmerksamkeit entgegengebracht. Art. 68 Abs. 3 des Römischen Statuts räumt erstmalig in der Geschichte der Völkerstrafgerichtsbarkeit den Betroffenen von Straftaten über eine passive Zeugenaussage hinaus eine aktive Rolle im Verfahren ein. Voraussetzung ist hier, dass das Römische Statut begrifflich zwischen Zeugen und Opfern unterscheidet. $^{32}$ Dies ist nicht nur aus viktimologischer Sicht als progressiv einzustufen, weil den Betroffenen die Möglichkeit gegeben wird, als selbstbestimmte Individuen und nicht bloß als Informationslieferant_innen innerhalb eines Verfahrens, das sich, weil es in erster Linie der Ermittlung der Schuld der Täter_in gewidmet ist, unabhängig von ihren Bedürfnissen und Erwartungen vollzieht, aufzutreten. Die Partizipationsgewährung gibt vor allem Aufschluss über das Gerechtigkeitsverständnis des IStGH, nach dem Gerechtigkeit für Betroffene eher prozesshaften Charakter zu tragen und nicht allein einem ergebnisorientierten, statischem Konzept zu folgen scheint. Von Betroffenenseite dürften sich die größten Erwartungen mit den „Grundsätzen für die Wiedergutmachung“ in Art. 75 Abs. 1 des Römischen Statuts, die Entschädigung, Rückerstattung und Rehabilitierung für Opfer von makrokriminellen Taten vorsehen, verbinden. Die Integration des Wiedergutmachungsaspektes in das Statut des Internationalen Strafgerichtshofs wird im Einklang mit den Forschungsergebnissen der Viktimologie ebenfalls als bedeutender Fortschritt erachtet. Die Möglichkeit der Entschädigung hängt jedoch von einer Verurteilung ab, was angesichts der Selektivität der Ahndungen zu einer Ungleichbehandlung und damit zu einer Gefahr neuen Konfliktpotentials führen könnte. ${ }^{33}$

Das Hauptargument für die Wiedergutmachung in Form von (materieller) Entschädigung beruht auf der Auffassung, dass diese Geste zumindest hinsichtlich der offiziellen Anerkennung individuellen Leids den Geschädigten Genugtuung zu verschaffen vermag. Ein entscheidender Unterschied zur Vergeltungstheorie besteht hierbei darin, dass nicht die monetäre Retribution, also das quantitative ,Aufwiegen' des Unrechts, im Vordergrund steht. Vielmehr besteht die Wiedergutmachung gemäß der Vorstellung einer restaurativen Gerechtigkeit ${ }^{34}$ in der Rehabilitierung der geschädigten Person bzw. in der Wiederherstellung ihrer Würde. Hierbei wird dem angenommenen Wunsch von Betroffenen nach Wahrheit, der als elementarer Bestandteil des „opfergerechten“ Strafverfahrens ange- 
sehen wird, ${ }^{35}$ in Form einer umfangreichen Tataufklärung nachgekommen. Dies kann insbesondere der Verarbeitung der Viktimisierungserfahrung der Betroffenen förderlich sein. Im Zuge eines Wiedergutmachungsprozesses ist der/die Täter_in in besonderer Weise gezwungen, sich mit den Folgen des Verbrechens, das von ihr/ihm verübt wurde, zu beschäftigen und das konkrete Leid der Betroffenen wahrzunehmen. Das heißt, über die Bemessung des Strafmaßes in Abhängigkeit der Tat hinaus wird ein Prozess der Aufklärung über das Unrechtsgeschehen in Gang gesetzt, der das asymmetrische Verhältnis von Täter_innen und Betroffenen direkt betrifft. Diesem Aufklärungsprozess kommt daher eine besondere Bedeutung zu, die im gegenwärtigen Forschungsstand noch nicht genügend Beachtung findet. Wichtig ist hier klarzustellen, dass der Zweck eines betroffenengerechten Verfahrens explizit nicht in der Repression gegenüber Täter_innen besteht. Ein solcher Strafzweck trüge schließlich selbst menschenrechtsverletzenden Charakter. Vielmehr geht es darum, dass das Gerichtsverfahren als der geschützte Ort betrachtet wird, in dem Betroffene ihre Verletzungen derart thematisieren können, dass sie nicht nur bei den Gerechtigkeit gewährenden Instanzen, sondern bei den Täter_innen selbst Gehör finden.

Oberste Priorität des betroffenengerechten Strafverfahrens hat die Bewahrung der Geschädigten vor Sekundär- und Tertiärviktimisierungen. Unter Sekundärviktimisierung wird im Unterschied zur Erstviktimisierung durch die Verbrechenstat selbst (die sich u.a. in Form von physischen und psychischen Verletzungen, emotionalen Schädigungen sowie materiellen Einbußen einstellen kann $)^{36}$ das erneute „Opferwerden durch Fehlreaktion der sozialen Umwelt auf die primäre Viktimisierung “37 verstanden. Insbesondere im gerichtlichen $\mathrm{Zu}$ sammenhang können bestimmte Behandlungsweisen im Zuge von Strafverfahren dazu führen, dass Betroffene re-traumatisiert werden oder es zumindest zu einer Intensivierung und Ausweitung des bereits erlittenen Schadens kommt, sofern nicht auf die besonderen psychischen und physischen Umstände von Betroffenen Rücksicht genommen wird. Mit Tertiärviktimisierung bezeichnet man schließlich mögliche Persönlichkeitsveränderungen bei Betroffenen, die bereits den Opferstatus nachhaltig in das eigene Selbstbild integriert haben. ${ }^{38}$ Insbesondere für diese Personengruppe ist es umso wichtiger, dass ihnen eine entsprechend sensibilisierte Interaktionsweise seitens Gerichtsangehöriger und anderer offizieller Akteure zuteil wird. Defizite im Umgang mit Betroffenen bei Gerichtsangehörigen sowie besonders auch ein mangelhafter Zeug_innenschutz, der die Betroffenen nicht vor der Gefahr, zur Angriffsfläche für Aggressionen zu werden, bewahrt, gehören zu den Hauptursachen für Sekundär- und Tertiärviktimisierungen.

Zur Vermeidung von Folgeviktimisierungen gehört jedoch nicht zuletzt auch, den Betroffenen keine unrealistischen Vorstellungen über konkrete Erfolgsaussichten auf finanzielle Entschädigung zu geben. Da der IStGH ausschließlich die individuelle strafrechtliche Verantwortlichkeit von Täter_innen feststellt, ergibt sich z.B. keine Wiedergutmachungspflicht für Staaten. Dieser Umstand ist vor dem Hintergrund des kollektiven Tatzusammenhangs und der komplexen und z.T. ambivalenten Rolle, die staatliche Akteur_innen in Konfliktsituationen spielen, als nicht unproblematisch zu erachten, da auch hier die Gefahr besteht, dass in der Praxis, u.a. aufgrund ungeklärter Verantwortlichkeiten seitens neuer Funktionsträger_innen einer Postkonfliktgesellschaft, nur sehr ausgewählte Geschä- 
digte berücksichtigt werden können. ${ }^{39}$ Das bedeutet, dass effektive und dauer-

hafte Maßnahmen zur Rehabilitierung der Würde und Autonomie von Betroffenen auf der Grundlage von Entschädigung nicht zu erwarten sind, geschweige denn die strukturellen Probleme einer von Kernverbrechen betroffenen Gesellschaft, die bis weit in ihre sozialen, politischen und kulturellen Bereiche hineinreichen, überwunden werden könnten.

\section{Vergangenbeitsbewältigung als Strafzweck?}

In Bezug auf die Berücksichtigung von Betroffenenbelangen ist deshalb eine Klärung, inwiefern im Rahmen von Gerichtsverfahren die Asymmetrie zwischen Täter_innen und ihren Verbündeten auf der einen Seite und den Geschädigten auf der anderen im Rahmen von Gerichtsverfahren abgebaut werden kann, unabdingbar: Zum einen, um den individuell Betroffenen eine fortgesetzte Traumatisierung zu ersparen, und zum anderen, um die Weichen für eine gelingende Transformation der Postkonfliktgesellschaft zu stellen. Der Umstand, dass die Betroffenen während eines bewaffneten Konflikts staatlicherseits nicht geschützt wurden, kann zwar im Nachhinein weder durch die Bestrafung der/des Täter_in ,vergolten' noch durch eine Entschädigung der/des Betroffenen rückgängig gemacht werden. Die Signalwirkung von Strafprozessen im Sinne der edukativen Systemprävention, zu der wesentlich die öffentliche moralische und politische Verurteilung der Verbrechenstat gehört, erhält insbesondere hinsichtlich des Ziels der Beendigung von Straflosigkeit jedoch umso mehr Gewicht - für die einzelnen Betroffenen, aber auch für die Gesamtgesellschaft in der Postkonfliktphase. Den Strafzweck edukativer Systemprävention als Ausdruck arroganter Siegerjustiz oder rachegeleiteter Politik, die sich die Rolle der Stellvertreterin von Betroffenen anmaßt, zu deuten, verkennt daher die eigentliche Bedeutung der Bestrafung von Täter_innen für die Geschädigten. ${ }^{40}$ Auf eine einfache Formel gebracht, lässt sich über den Zusammenhang zwischen der Strafe für die/den Urheber_in der Verbrechenstat und der/dem durch sie Geschädigten sagen: Das „Verbrechensopfer hat von der Bestrafung des Täters nichts, erlitte aber viel, wenn es zu ihr nicht käme“. ${ }^{41}$ In den meisten Fällen verspüren Geschädigte nicht zuletzt, weil sie um die Nicht-Vergeltungsfähigkeit des erfahrenen Unrechts wissen - angesichts der Verurteilung von Täter_innen weniger eine auf die eigene Person bezogene Befriedigung, sondern vielmehr die Genugtuung, dass das Recht durch ein Strafverfahren restituiert wird. ${ }^{42}$ „I've come to see that justice is done“ - so wird die Zeugin „FWS-75“ im Fall Kunarac und andere vor dem JStGH in der Studie von Kathrin Greve zitiert, in der u.a. untersucht wird, welchen Beitrag Zeuginnenaussagen in internationalen Strafprozessen bei der Auf-

Insbesondere die Legitimität des Wunsches eines Opfers nach Genugtuung, zu dessen Befriedigung sogar Vergeltung i.S.d. absoluten Strafzwecks ins Spiel gebracht wird, steht im Focus der Skepsis, ob die Einbeziehung von Betroffenenbelangen einem "humanen Strafrecht“ eventuell zuwider zu laufen drohe, vgl. Jäger (Fn. 4), 271; Reese (Fn. 1), 83. Betrachtet man allerdings die Gesamtheit der Strafzwecke in der internationalen Völkerstrafjustiz, erweist sich die Befürchtung, dass eine repressive Justiz wichtige Limitierungen völkerrechtlicher Strafgerechtigkeit bzw. menschenrechtliche Schutzansprüche der/des Täter_in unterminiere, als unbegründet: Gemäß Römischem Statut ist die Todesstrafe ausgeschlossen, es gilt prinzipiell die Unschuldsvermutung (vgl. Art. 66), der Bestimmtheitsgrundsatz sowie das Doppelbestrafungs- und das Rückwirkungsverbot (vgl. Art. 20-24). Zudem wird in Art. 67 eine Reihe von Rechten der/des Angeklagten aufgeführt, die sich auf den fairen Umgang mit ihrer/seiner Person während des Verfahrens beziehen.

Hassemer/Reemtsma (Fn. 7), 138.

Vgl. Hassemer/Reemtsma (Fn: 7), 146. 
arbeitung von sexualisierter Gewalt in Konfliktsituationen leisten. ${ }^{43}$ Angesichts der durchaus realistischen Einsicht, dass weder einzelne Betroffene noch die Postkonfliktgesellschaft im Ganzen „mit einem Menschheitsverbrechen wirklich vollständig und lückenlos ,fertig werden'“44 kann, ist daher zu fragen, welche Plausibilität der Strafzweck edukativer Systemprävention beanspruchen kann. Damit soll nicht der gängige und durchaus gerechtfertigte Vorwurf, dass die juristische Praxis an internationalen Strafgerichtshöfen - nicht zuletzt aufgrund knapper Ressourcen - von politischer Selektivität gekennzeichnet ist, ${ }^{45}$ bedient werden. Solche praktischen Defizite und Hindernisse erschweren zweifellos die politische und rechtliche Transformation einstiger Konfliktgesellschaften. Doch ruht hier das Augenmerk vielmehr auf dem normativen Anspruch, Vergangenheitsbewältigung (im Rahmen z.B. der edukativen Systemprävention) als Strafzweck des Völkerrechts zu deklarieren, mit dem sich die Hoffnung zu verknüpfen scheint, dass mit der juristischen Behandlung von Unrechtstaten die Bewältigung der Verbrechen regelrecht ,abgeleistet' werden.

\section{Probleme einer auf juristische Vergangenheitsbewältigung ausgerichteten Völkerstrafgerechtigkeit}

Die eigentliche Herausforderung des Völkerstrafrechts hinsichtlich einer effektiven Berücksichtigung von Betroffenenbelangen besteht hingegen in etwas gänzlich Anderem, das die kroatische Schriftstellerin Svetlana Drakulić, die als Prozessberichterstatterin in Den Haag tätig war, folgendermaßen auf den Punkt bringt: „Die Prozesse gegen die Kriegsverbrecher sind nicht nur wegen der Toten wichtig, sondern auch wegen der Überlebenden. Denn schließlich muß sich jeder einzelne fragen: Was hätte ich in so einer Situation gemacht? “46 Diese Frage verweist mithin auf ein Problem, das nicht nur in Bezug auf Postkonfliktgesellschaften, sondern allgemein gilt: Kernverbrechen unterscheiden sich hinsichtlich ihres Schweregrads und Ausmaßes zwar durchaus faktisch von „alltäglicher“ Kriminalität. Doch besteht die Gefahr des Scheiterns von zivilisierten Gesellschaften immer und überall latent. Es sind nicht ausschließlich die apostrophierten totalitären Systeme, die makrokriminelle Täter_innen hervorbringen. ${ }^{47}$ Aus dieser Annahme schlussfolgert etwa Vasco Reuss, dass gerade hinsichtlich der Berücksichtigung von Betroffenenbelangen der Aspekt edukativer Prophylaxe umso dringlicher bei der Ausgestaltung des Strafzwecks der Systemprävention zu berücksichtigen sei. Durch das Völkerstrafrecht müsse verdeutlicht werden, dass selbst im Falle staatlicher Unrechtstrukturen die Gültigkeit von Menschenrechten weiterhin bestehen bleibt - was wiederum bedeutet, dass für die Geltung der Menschenrechte im Konfliktsfall die/der potenzielle Täter_in selbst verantwortlich ist. Das Völkerstrafrecht böte nach dieser Auffassung mittels einer Spezifizierung der Definition des generalpräventiven Strafzwecks im Sinne einer menschenrechtsbasierten Erziehung zur Zivilcourage die Möglichkeit, nicht nur die Geltung des Rechts, sondern darüber hinaus die Gültigkeit einer umfassenderen Gerechtigkeit, die auf universalen Menschenrechten beruht, zu bekräftigen. Plausibel an Reuss‘ Ansatz erscheint daher genau dieser auf die Förderung

Vgl. Greve, Vergewaltigung als Völkermord. Aufklärung sexueller Gewalt gegen Frauen in internationalen Strafgerichten, Baden-Baden 2008, 1. Zum Fall s. Prosecutor v. Dragoljub Kunarac and others, Judgment, IT-96-23-T \& IT-96-23/1-T (22.2.2001). 
eines allgemeines Menschenrechtsbewusstseins abzielende Aspekt: Andernfalls

nämlich ergibt die Zuschreibung individueller Verantwortung von Täter_innen und die damit verbundene Kriminalisierung, die ja gerade als Fortschritt des Völkerstrafrechts angesehen wird, keinen Sinn.

\section{Für die Justiz allein eine Nummer zu groß. Ein Fazit}

Die Bewältigung begangenen Unrechts ebenso wie die erzieherische Prophylaxe sind sicherlich als begrüßenswerte Schritte in Richtung einer die Belange von Betroffenen berücksichtigenden Zielsetzung des Völkerstrafrechts zu werten. $\mathrm{Ob}$ die Förderung von gesellschaftlicher Zivilcourage ein originärer Strafzweck sein kann, ist allerdings zu bezweifeln. Der Verdacht, dass mit dem Strafzweck der Zivilcourageförderung die Kapazität des Völkerstrafrechts heillos überfordert würde, lässt sich erhärten, wenn man überlegt, das die konkrete Umsetzung des Ziels der Erweiterung einer zivilcouragierten Grundhaltung in der Bevölkerung Ressourcen und Maßnahmen erfordert, die weit außerhalb der spezifischen Aktions- und Wirkungsmöglichkeiten der Justiz liegen. Im Gegensatz zu Reuss Vorstellung einer Übernahme des Zivilcourage-Gedankens in die Strafzwecksetzung des Völkerstrafrechts halte ich es für sinnvoller, dass Vergangenheitsbewältigung und Förderung von Zivilcourage als übergeordnete Ziele institutioneller und individueller Bemühungen um die gesellschaftliche Aufarbeitung von Unrechtstaten in Konfliktgesellschaften verstanden werden sollten, zu deren Erfüllung die juridische Arbeit ihren spezifischen, gleichwohl unentbehrlichen und fundamentalen Beitrag leistet. Der Justiz kommt dann hinsichtlich einer umfassenderen, die gesamte Gesellschaft in der Postkonfliktphase und ihre sozialen und politischen Institutionen mit einbeziehenden Aufarbeitung zwar eine durchaus begrenzte Rolle zu. Doch sollte der Wirksamkeitsradius der Beendigung von Straflosigkeit nicht unterschätzt werden. Die Ergebnisse der Strafgerichtsverfahren vor internationalen Gerichten, und genau darauf zielt die edukative Systemprävention ab, verfügen über eine unverzichtbare normative Ausstrahlungskraft, weil sie auf der Basis von aufklärender Ermittlungsarbeit zu den Kernverbrechen, den Hintergründen ihrer Entstehung sowie ihren Folgen für die Betroffenen die Zuschreibung von individueller Verantwortlichkeit der Täter_innen ermöglichen. Damit erreicht die juristische Aufarbeitungsarbeit eine dezidierte Normverdeutlichung, was von entscheidender Bedeutung für eine Gesellschaft ist, die auf einer rechtsstaatlichen Ordnung gründen soll - diese kann ausschließlich im Rabmen von Rechtsprechungsprozessen konstituiert bzw. konsolidiert werden.

Über den engen Rahmen der Strafgerechtigkeit hinausgehend, ${ }^{48}$ kommen dann erprobte zivilgesellschaftliche Interaktionsmodi zwischen Betroffenen und Täter_innen in öffentlichen gesellschaftlichen Foren - z.B. in Wahrheits- und Versöhnungskommissionen mit ihren Praktiken der wechselseitigen Aussprache, symbolischen Formen von Entschuldigung und anderen Varianten einer Kommunikation der Anerkennung - zum Tragen. ${ }^{49}$ Diese Maßnahmen stellen die zentrale Bedeutung strafrechtlicher Sanktionierung nicht in Frage. Im Gegenteil, sie fungieren als Begleitinstrumente des institutionellen Wiederherstellungspronungsprozess, in: Buckley-Zistel/Kater (Hrsg.), Nach Krieg, Gewalt und Repression. Vom schwierigen Umgang mit der Vergangenheit, Baden-Baden 2011, 81-90.

49 Vgl. Hitzel-Cassagnes/Martinsen, Zum Verhältnis von sozialer Anerkennung und Rechtsprinzip im Rahmen der Bewältigung historischen Unrechts: Konkurrenz oder Komplementarität?, in: Schramm/ Frey/Kähler/Müller-Mall/Wapler (Hrsg.), Konflikte im Recht - Recht der Konflikte, Archiv für Rechts- und Sozialphilosophie, Beiheft 125, Baden-Baden 2010,273-294. 
zesses von Recht und Gerechtigkeit. Insbesondere letztere würde dementsprechend weniger als das exakt definierte Ergebnis einer an der Erfüllung von $\mathrm{Pa}$ ragraphenvorgaben orientierten Justiz, sondern im Wesentlichen als Ermöglichung der dynamischen Wiedergewinnung von Würde und Autonomie für die ehemaligen „Opfer“ aufgefasst werden können. Die Erlangung von Gerechtigkeit im Sinne von Autonomie für Betroffene sieht sich allerdings mit einer nicht unerheblichen Bedrohung konfrontiert, sofern das Ziel der Verfahren vor dem IStGH in erster Linie der Wahrung des Weltfriedens dient, dem die Belange von Individuen nachgeordnet werden. ${ }^{50}$

\section{Chancengleichheit}

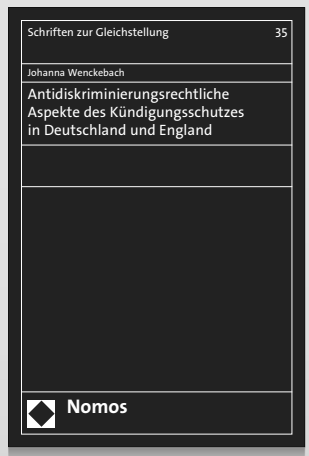

\section{Antidiskriminierungsrechtliche Aspekte des Kündigungsschutzes in Deutschland und England}

Von Dr. Johanna Wenckebach

2012, 367 S., brosch., 84,- $€$

ISBN 978-3-8329-7683-5

(Schriften zur Gleichstellung, Bd. 35)

Für die praxisrelevanten Gleichheitsfragen im Bereich des Kündigungsschutzes bietet das in dieser Studie untersuchte, gleichheitsrechtlich geprägte Arbeitsrecht Englands umfangreiches Anschauungsmaterial und Lösungsmöglichkeiten. Mit der Analyse der europarechtlichen Vorgaben wird ein wichtiger Beitrag zur Debatte um Chancengleichheit im Arbeitsleben geleistet. 